Madalina Akli est candidate au doctorat en études françaiseà la Rice University (Houston, Texas).

\title{
Exode vers des terres sèches
}

Depuis douze heures, elle reste patiemment assise dans le siège de la voiture. Elle est fatiguée, mais calme. Son corps ne sent presque plus rien, pas de faim, pas de soif, pas d'envie de bouger. Les voitures autour avancent à peine, et depuis hier soir, ce cordon compacte sur l'autoroute refuse de s'espacer. Le trafic est lourd - quatre voix dans le même sens et ça ne suffit pas. Tout le monde veut aller vers le nord, mais voilà, puisque le nord est tant convoité, il faut bien être patient! Et du temps, il y en a! Même en n'avançant que quelques mètres par heures, en vingt-quatre heures, tout le monde a l'espoir de bien arriver au nord.

Elle est là, dans sa voiture, au milieu d'une masse mouvante, dirigée dans le même sens. "C'était la bonne décision! ", se dit-elle. Tous dans la même direction - l'esprit de troupeau est bien rassurant. Et tous dans les mêmes conditions : en voiture, avec les enfants, les valises, le chien, l'ordinateur... C'est comme dans une maison mobile. Personne ne pense plus à l'autre maison, la vraie. Entourée par cette foule-famille, elle se rassure encore une fois : " Oui, c'était la bonne décision. " De toute façon, elle ne sait pas nager. Alors, mieux d'être au sec que dans Dieu sait quelle ville aquatique.

Cette voiture-maison est vraiment confortable. Et sûre aussi : elle est toute neuve, elle ne fait pas de bruit et, en marche depuis douze heures, son petit cour n'arrête pas de battre dans un rythme qui fait penser à une personne en pleine santé. Aucun essoufflement, aucune émotion inutile. Oui, c'est bien d'être dans le corps de cette voiture qui se porte si bien. Tant qu'elle ne fait pas de problèmes, tout va bien. Et pourquoi ferait-elle des problèmes? Il n'y a pas la moindre chance, ou plutôt malchance, que quelque chose puisse arriver à cette voiture, que quelqu'un ou quelque chose pourrait perturber le rythme ininterrompu de son petit cœur.

Si elle avait marché à pied, elle aurait peut-être devancé la voiture d'une bonne dizaine de kilomètres. Eh, il ne faut pas faire la tête; il vaut mieux faire corps avec la voiture, en particulier quand un ouragan de force cinq annonce sa visite. Bientôt, quatre voies de plus seront ouvertes, et alors, aucune suspicion que cette petite bagnole pourrait être rattrapée. «De la patience, juste de la patience, et cette Honda va s'occuper du 
reste! " Elle va bien arriver au nord; elle va bien s'en sortir, il n'y a pas de doute. Mais où sont ces quatre voies supplémentaires? C'est vrai qu'elle ne peut pas faire grand-chose si elle ne peut pas rouler. Allez, go, go!

Une petite onde de douleur aigue s'installe au-dessus de l'oeil gauche, proche de la tempe. Elle palpite nerveusement, l'espace de quelques secondes, et après elle disparaît comme si rien n'était. Mais quand même, à force de se répéter, l'induction de cette onde a fini par se faire attendre. C'est comme une aiguille qui pique le cerveau, et après avoir laissé son empreinte, se retire en hâte sans laisser de trace. Ça doit être la fatigue qui s'accumule. Maintenant, ça fait dix-huit heures depuis qu'elle est sur la route. Il serait vraiment temps que ces quatre voies supplémentaires s'ouvrent.

Le calme autour est un peu angoissant. D'ailleurs, on dirait que c'est seulement la vitesse lente des voitures qui contribue à ce calme de plus en plus extérieur. Car à l'intérieur, souvent, les gens tournent nerveusement la tête à gauche, vers ces quatre voies complètement vides et promises à l'ouverture en contresens, depuis bientôt six heures. Il faut s'éloigner au nord, et pour ce faire, il faut quand même bouger un peu.

La douleur revient dans la tempe gauche et se renouvelle les forces. Elle entre comme un marteau-piqueur et touche les profondeurs de l'oreille. Son corps commence à se réveiller. Les doigts tremblent un peu, comme dans un mouvement nerveux d'intensité moyenne. Mais il faut que ce mouvement cesse, car il n'a aucune autorisation de se manifester, il n'a ni même aucune raison d'être.

Pour l'amour de Dieu, cette autoroute devrait s'élargir le plus tôt possible, puisque sinon l'ouragan va bientôt finir par faire la connaissance à cette foule de gens, ici même sur l'autoroute!

L'indicateur d'essence est à moitié. Des voitures autour sont déjà une panne d'essence, tout comme les stations sur toute la distance du bouchon. La voiture, d'accord, elle est l'arche de Noé, mais pas sans essence, ça c'est sûr!

L'Honda continue imperturbablement le même rythme depuis le début. Quoi, elle ne va pas crever comme ça, sans aucun signe de dysfonctionnement. Elle ne va pas tout simplement s'arrêter!

Chaque ligne de l'indicateur d'essence qui descend a un effet de connexion à cette douleur emmerdeuse qui se plaît à descendre de plus en plus de l'oreille vers l'omoplate. Elle en sort et elle y rentre méthodiquement, à l'intervalle égal, correspondant à chaque retombée successive de l'indicateur de l'essence. Oh non, ça ne peut pas se terminer comme ça! Cette voiture toute neuve, ce bébé en bonne santé ne va pas tout simplement s'arrêter de vivre. D'accord, la vie est absurde, mais pas à ce point-là, surtout 
pas à ce moment-ci. Dites-lui qu'elle ne peut pas s'arrêter tout court, à cause de manque d'essence, alors que depuis des heures elle roule si bien. Mais non, elle n'a aucun problème! Bref, l'essence, et alors quoi, elle doit ne plus respirer? Justement, maintenant quand il y a de l'air et surtout de l'espace... car l'autoroute vient de s'élargir!

Les larmes montent aux yeux. Oui, classique! Sauf que maintenant elle sent que ça a l'air d'être pour la dernière fois. Oh, pathétique! Mais voilà, elle sait qu'elle ne va pas pouvoir arriver au nord et d'un coup, les vingtquatre heures prévues pour l'évacuation deviennent comme ça, l'air de rien, les dernières vingt-quatre heures de la vie. " Mais laisse, lui dit-il, on va bien se sauver. On va chercher abri dans un bâtiment. "Elle aimerait bien le croire, c'est juste que se voir expulser de cette voiture-salut vers un total imprévisible, elle ne peut pas l'assimiler. Pourtant elle essaie : c'est simple, de cette voiture, de toute façon trop petite, elle peut aller dans un quelconque bâtiment, plus grand, plus sûr. Il n'est pas possible d'arriver au nord. Elle se tortille dans le siège de la voiture, les mains tremblant, les yeux protubérants : "Oui, oui c'est ça, c'est bien ça, on va aller chercher abri dans un bâtiment. » 